\title{
A systematic evaluation of accessibility measures by the two- step floating catchment area (2SFCA) method
}

\author{
Xiang Chen ${ }^{\mathrm{a},} *$, Pengfei Jia ${ }^{\mathrm{b}}$
}

${ }^{a}$ Department of Geography, University of Connecticut, Storrs, Connecticut 06269, USA, chenxiangpeter@ gmail.com

${ }^{b}$ China Academy of Urban Planning and Design, Beijing, 100044, China, j_pengfei@yahoo.com

* Corresponding author

Keywords: Accessibility, Spatial interaction model, 2SFCA

\begin{abstract}
:
Accessibility, as an important theme in geospatial science, measures the potential of interaction between geographic entities. Originated in Hansen's (1959) empirical model for land use planning, place-based accessibility becomes an integrated assessment of urban settlements in relation to social services and opportunities, such as employment, education, and entertainment. Traditional place-based accessibility models, such as the integral measure or the cumulative-opportunity measure (Kwan, 1998), are primarily dependent on the assessment of the supply (e.g., stores, restaurants), evaluating if goods or services could be delivered or reached at an acceptable cost (e.g., distance, time). This assessment overlooks the complex spatial interactions between the supply and demand, referred to as the "complementarity" (Haynes \& Fotheringham, 1984). Recent development of the place-based accessibility theory revolves around the two-step floating catchment area (2SFCA) method (Luo \& Wang, 2003). The model evaluates if the capacity of service facilities can cater to nearby demand in a two-step search process. Initially serving for the assessment of health care facilities, the model has been further modified to accommodate various urban planning scenarios (Chen, 2017).

One compartment of the model in need of further evaluation is the distance decay. Although the 2SFCA model and its extensions have involved different distance decay functions, such as the Gaussian form and the kernel density form, there is a limited scope of work systematically comparing the performance and limitations of different 2SFCA models. In this study, we have proposed an analytic framework that includes six distance decay functions: the rectangular cumulative-opportunity (CUMR), negative-linear cumulative-opportunity (CUML), inverse-power gravity-type (POW), exponential gravity-type (EXP), and Gaussian gravity-type (GAUSS), and kernel density (KD) models. Examples of these distance decay functions are shown in Figure 1. Each model further consists of four variable scenarios, generating a total of twenty-four 2SFCA measures for comparison in a systematic manner.
\end{abstract}

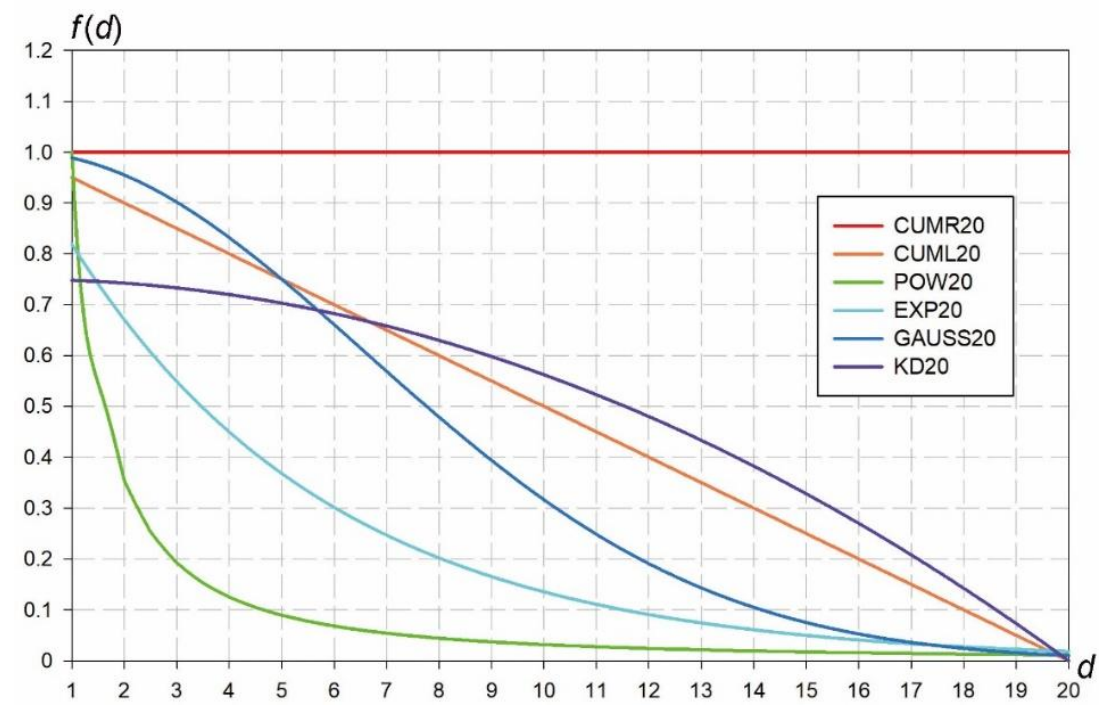

Figure. 1. Illustration of different distance decay functions in the 2SFCA model.

Using the datasets of point-based food stores (i.e., the supply) and population (i.e., the demand) in the state of Arkansas, the United States, three sets of sensitivity analyses have been conducted to compare the results derived from these twenty-four models. These analyses include (1) Pearson's correlation between models, (2) assessment by urban-rural status, and (3) variability analysis of the catchment size. Observations about the sensitivity of the 2SFCA models to the distance decay function and the catchment size are drawn from the analyses, providing valuable information for better understanding the intricacy of the model compartments. For example, we have employed the coefficient of variation 
$\left(C_{\mathrm{V}}\right)$, defined as the division of the standard deviation to the mean, to examine the spatial inequity of different $2 \mathrm{SFCA}$ models as a function of the catchment size $\left(d_{0}\right.$, in miles). As shown in Figure 2, all models have a large degree of variability with a small $d_{0}$; when $d_{0}$ increases to a certain threshold, $C_{\mathrm{v}}$ becomes relatively convergent $\left(d_{0} \geq 9.5\right)$. It is also observed that POW20 has a higher level of variability than other models. In this respect, POW20 should be avoided in future model implementation as it derives a different spatial inequity pattern than other models.

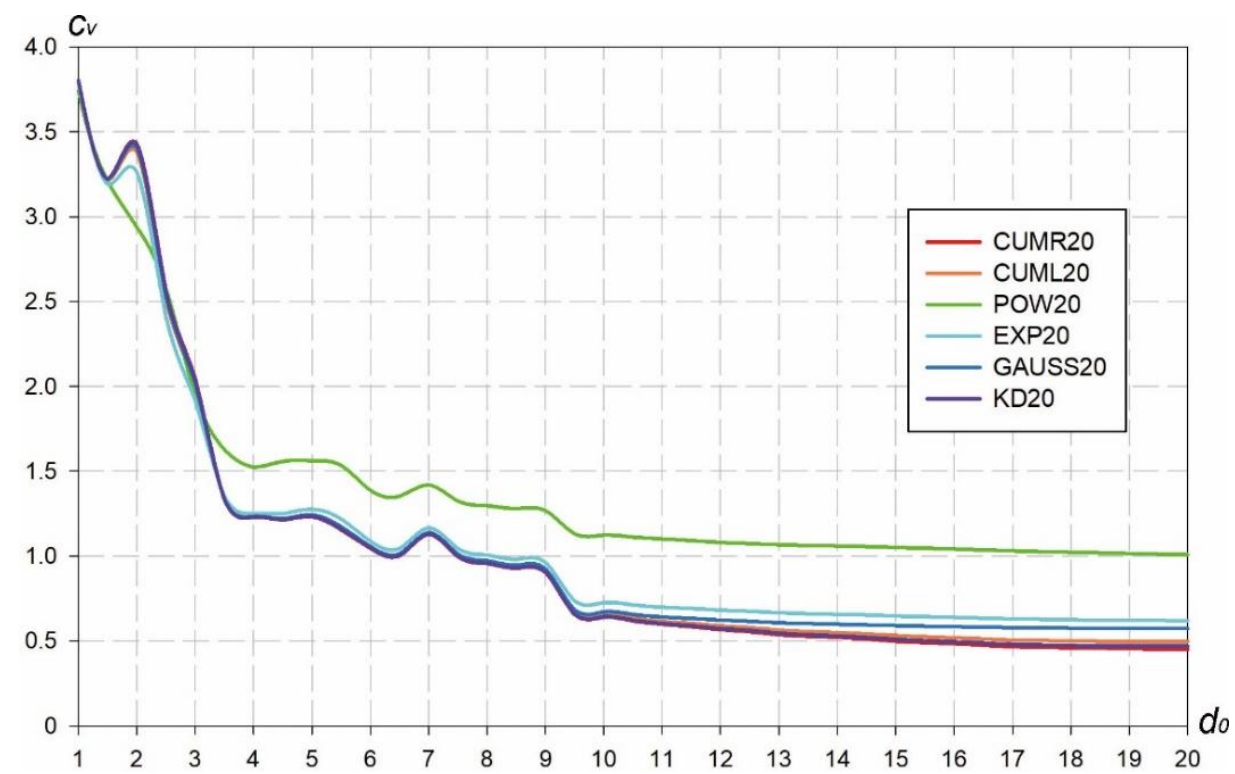

Figure 2. Coefficients of variation $\left(C_{\mathrm{V}}\right)$ of different 2 SFCA models as the catchment size $\left(d_{0}\right.$, in miles $)$ increases.

In addition to revealing the applicability of the models, the paper further draws two important conclusions. First, on a small analysis scale (e.g., community), the catchment size is the most important modeling variable. In this scenario, variation in the catchment size can cause a high degree of measurement uncertainties. Thus, it is a necessity to examine and justify the choice of the catchment size when applying the 2SFCA model to a small-scale analysis. Second, on a large analysis scale (e.g., state, province), the distance decay function is of critical importance. In this scenario, using the 2SFCA model without the distance decay will likely overestimate the supply-demand interaction and thus obfuscate the inequity pattern. In sum, the comparison and the sensitivity analysis outline the potential applicability and limitations of different 2SFCA models. It provides the theoretical rapport necessary to future applications of the model for various urban planning, service delivery, and spatial equity problems.

\section{References}

Chen, X. (2017). Take the edge off: A hybrid geographic food access measure. Applied Geography, 87, $149-159$.

Haynes, K. E., Fotheringham, A. S. (1984). Gravity and spatial interaction models (Vol. 2). Beverly Hills: Sage Publications.

Hansen, W. G. (1959). How accessibility shapes land use. Journal of the American Institute of Planners, 25(2), 73-76.

Kwan, M. (1998). Space-time and integral measures of individual accessibility: A comparative analysis using a pointbased framework. Geographical Analysis, 30(3), 191-216.

Luo, W., Wang, F. (2003). Measures of spatial accessibility to health care in a GIS environment: Synthesis and a case study in the Chicago region. Environment and Planning B: Planning and Design, 30(6), 865-884. 